\title{
Prospects of Perineural Implantation of Stem Cells in Recovery of Neural Network's Functions in Brain Diseases
}

\author{
Kulchitsky Vladimir*1, Zamaro Alexandra ${ }^{1}$, Shanko Yuri $^{2}$ and Koulchitsky Stanislav ${ }^{3}$ \\ ${ }^{1}$ Institute of Physiology, National Academy of Sciences, Minsk, Belarus \\ ${ }^{2}$ Republican Scientific and Practical Center of Neurology and Neurosurgery, Minsk, Belarus \\ ${ }^{3}$ Liege University, Liege, Belgium
}

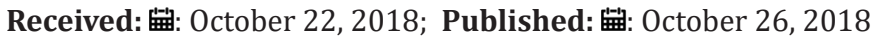

*Corresponding author: Kulchitsky Vladimir, Institute of Physiology, National Academy of Sciences, Minsk, Belarus, Europe

\begin{abstract}
Background: The question on prospects of additional use of cellular therapy in standard protocols of brain neural networks recovery after neurodestructive processes of various etiology was analyzed. Data on low efficiency of existing methods of neurodestructive processes treatment gave a boost to authors to conduct series of studies related to brain diseases and cellular technologies. Existing treatment principles now should be updated with new methods - in particular, cellular technologies. This statement was based on stem cells (SC) presence in human brain. Nature has formed basis for neural network formation in the process of training and for recovery therapy in case of pathology. Unfortunately, recovery potential of brain SC appeared to be ineffective in neurodestructive processes. Therefore, SC should be additionally injected into brain. It is advisable to use autologous SC in order to avoid possible side effects, such as malignization of allogeneic and other SC. But in certain cases, use of allogeneic SC can be approved.
\end{abstract}

Methods: Autologous mesenchymal SC (MSC) are usually administered to patients in experimental and clinical conditions. Two ways of MSC delivery to brain are preferred: with cerebral blood flow after MSC injection into bloodstream and MSC implantation close to damaged area after additional skull trepanation. Authors experimentally developed and clinically evaluated new technique of perineural implantation of MSC. This method is based on natural ability of SC to migrate. MSC are implanted into the area of cranial nerve endings (mainly olfactory and trigeminal) and then they migrate along cranial nerves to cranial cavity.

Results: The area of vomeronasal complex (VC) is preferred for MSC implantation because its mucosa has nerve endings of both olfactory and trigeminal nerves. Such technique of MSC implantation was substantiated by authors and was named perineural implantation of MSC when used in neurodestructive processes.

Discussion and Conclusion: The article contains critical analysis and prospects of cellular technologies development for therapy of brain diseases. Special attention is paid in discussion to technology of perineural implantation of MSC in experimental and clinical conditions.

Keywords: Neurodestructive Processes; Stem Cells; Mesenchymal Stem Cells; Reparation of Neural Network Functions; Recovery of Brain Functions Control; Vomeronasal Complex

Abbreviations: SC: Stem Cells; MSC: Mesenchymal Stem Cells; VC: Vomeronasal Complex

\section{Introduction}

Search for various logical combinations for SC and brain diseases in PubMed on October 19, 2018 showed 29934 articles about "brain injury cerebrovascular diseases", 764 about "brain injury cerebrovascular diseases stem cells", 414 about "brain injury cerebrovascular diseases stem cells human" and 4 - about "brain injury cerebrovascular diseases stem cells human intranasal".
Surprisingly, but these four articles contain information about administration of human SC to experimental animals, but not about therapy of human brain diseases. Authors of one article [1] used human MSC taken from umbilical cord of newborn babies for intranasal application in rodents with previously simulated hypoxia or brain ischemia. It was established that intranasal 
administration of human embryonic neural SC to neonatal rats with signs of encephalopathy is accompanied with more effective and rapid recovery of behavioral reactions control [2]. Intranasal injection of MSC from umbilical cord to Wistar rats is accompanied with activation of microglia and astrocytes leading to appearance of reparative properties of oligodendrocytes [3]. This process is also accompanied with acceleration of myelinization of neuron processes and optimization of interneuronal communications together with reduction of gliosis zone in damaged brain regions [3]. The range of mechanisms of positive effects of C3a peptide after its intranasal application to rodents was specified [4]. Therefore, there are no articles in PubMed related to intranasal administration of autologous SC for therapy of patients with brain diseases.

Authors started conducting studies for assessment of SC perineural implantation significance in activation of reparative processes about 10 years ago [5-12]. Patterns of SC migration in brain tissue have been established in the experiments - depending on the area of SC injection at cranial nerve peripheral endings and localization of neurodestruction site in brain tissue [5,10-12]. Such principle of migration was determined as somatotopic distribution of SC in brain [12].

SC consistently move directly to the site of destruction in brain [5-12]. It is already proved nowadays that such aimed migration is determined by various signaling molecules which are expressed by nerve and glial cells in the area of neurodestruction [5-12]. Somatotopic distribution of implanted SC in brain depending on the site of injection is another one regularity [12]. In particular, implantation of SC into the area of olfactory nerve endings results in SC distribution mainly in anterior and middle cranial fossae. Implantation of SC into the area of trigeminal nerve endings results in SC accumulation in posterior cranial fossa [12]. Additionally, significance of the amount of implanted SC was experimentally verified: it should be from several tens of thousands up to millions per one ml of culture medium. It was also experimentally stated that the best reparative results are reached when SC are implanted during first hours and days of neurodestruction development $[8,11,12]$.

\section{Modern Technologies of Brain Diseases Therapy}

World statistics characterizes low efficiency of diagnostics and treatment of acute and chronic brain diseases $[13,14]$. The situation initiates search for new more effective technologies to resolve socially important issues affecting aspects of life and capability of citizens around the world. About six million people die due to stroke with various parts of blood vessels involved [13] each year according to WHO $[13,14]$. About 10 million people die each year due to brain trauma. Surgery remains one of the key methods in treatment of such fatal cerebral diseases as stroke, brain trauma, cerebral aneurysms and neoplasms. Effectiveness of surgery really increases with implementation of robotic devices (da Vinci Surgical System, Spine Assist, Renaissance Robotic Systems), hightech operations (micro-, endovascular and stereotaxic surgery), combined therapy and new methods of rehabilitation [13], creation of electronic and other devices, some of them have already helped
Stephen Hawking realize his unique intellectual dispositions. Possibilities of diagnostic procedures in cerebral diseases also enhance due to modern electronic equipment (CT, MRI, PET). These technologies improve diagnostics at early stages of brain diseases, but, unfortunately, don't improve treatment results [13].

All the countries constantly perform search for new more effective ways of brain diseases treatment. And these methods have been already developed in the fields of cellular biology and neurophysiology. For example, cellular therapy was successfully adapted for treatment of socially important diseases [8,9,11-14]. There were both followers and opponents of their implementation in clinical practice - this usually happens when elements of novelty appear in science and technics. Authors overcame that stage and moved from experimental studies to clinical implementation. This refers to technique of perineural implantation of SC $[11,12]$, which has set of advantages compared to traditional systemic applications of SC (intravenous, intraarterial and intrathecal) [13-19]. Enthusiasts mastered hard period of substantiation of prospects of cellular technologies use in therapy of brain diseases. In fact, we have new stage nowadays when experimental studies are rapidly followed by implementation of new methods to clinical practice. There are both encouraging $[11,15,17-19]$ and negative $[8,12,16,20]$ results of cellular technologies use by doctors. It was found that intravenously and intraarterially implanted SC have extremely low ability to penetrate through blood-brain barrier to brain tissue $[15,18]$. There is another complication after intrathecal injection of SC: cells are unable to cope with craniocaudal flow of liquor [12]. Neurosurgeons perform SC implantation directly to brain tissue, but these manipulations are associated with additional surgical intervention (skull trepanation) which is undesirable in acute period of disease development [17]. The technique of perineural MSC migration of MSC to brain appeared to be an original way out $[7,8,11,12,20,21]$ in contrast to traditional surgical interventions. Enhancement of this technique allowed developing somatotopic method of MSC implantation followed by their aimed migration to certain brain region $[8,12]$ together with ways of visualization of migration process in clinical conditions [22-25]. Implantation of MSC was performed into the VC area (Figure 1) from where MSC migrate to region of neurodestruction by both olfactory and trigeminal nerve endings [22-25].

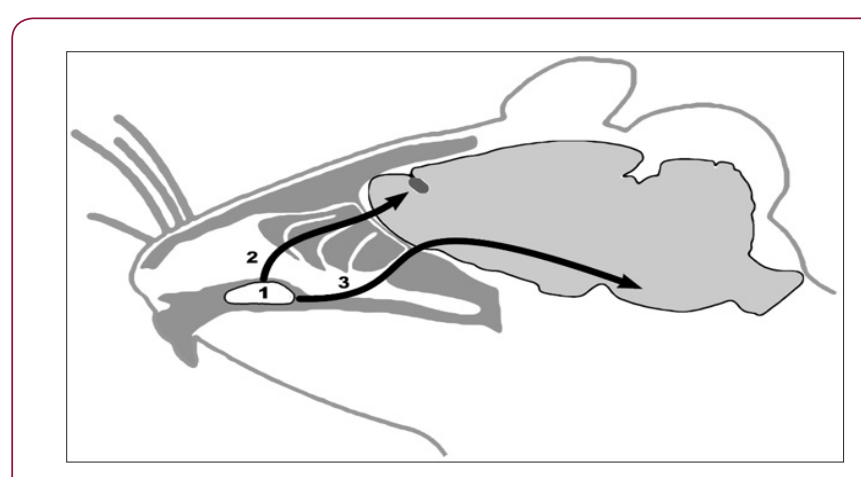

Figure 1: Hypothetic ways of SC migration from VC of rat (1) along olfactory (2) or trigeminal (3) nerves to cranial cavity (Figure by Dmitry Tokalchik). 


\section{Material and Methods}

Technique of MSC implantation into nasal submucosa was chosen due to minimization of side effects and simplicity of accomplishment by neurosurgeon [8,12,20-22]. Migratory abilities of SC were taken into account during technique development $[5,6,8,9,11,12]$. Moreover, the presence of endogenous SC in nasal mucosa says for MSC implantation namely in that receptive field [8-11]. Considering localization of central structures of olfactory analyzer in anterior cranial fossa, it was proposed that the bulk of implanted SC will be located namely in the structures of anterior cranial fossa.

Adipose tissue was previously separated from epiploon of anesthetized rats, fermented with trypsin and plated in plastic flasks with culture medium and gentamycin. MSC were painted for monoclonal antibodies to CD90 (FITC- or PKH67-labeled) at the day of implantation. Brain slices $8 \mu \mathrm{m}$ in thickness were prepared and examined with fluorescent microscope Zeiss Axio Vert 200M at the final stage. Concentration of MSC in experiments on rats was 50 thousand cells per $50 \mu \mathrm{l}$ of culture medium. Concentration of MSC for intranasal implantation in patients was from 6 to 10 million cells per $1 \mathrm{ml}$ of culture medium.

\section{Results}

Figure 2 shows distribution of FITC-labeled MSC in the area of lamina cribrosa ethmoid bone of rat after preliminary local destruction of brain in sensorimotor zone and subsequent implantation of 50 thousand MSC in $50 \mu \mathrm{l}$ of culture medium in the VC region.

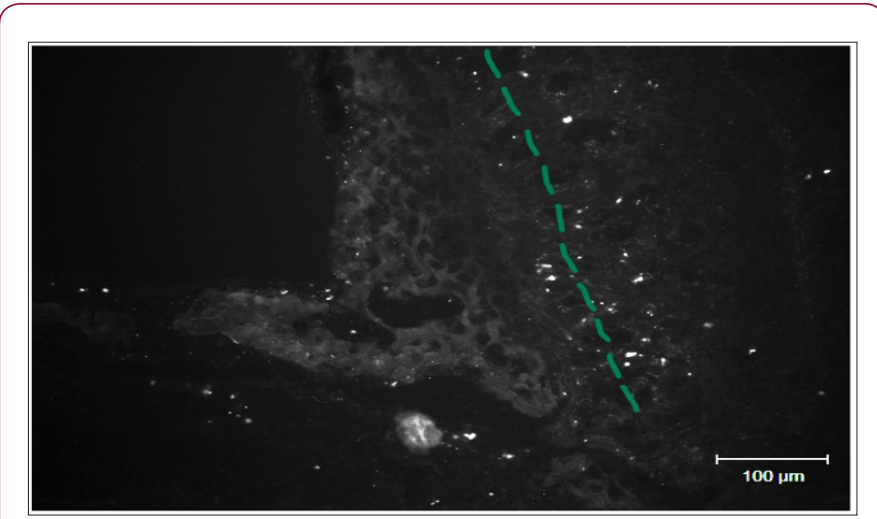

Figure 2: A line represents mesenchymal stem cells migration pathways in the area of lamina cribrosa ethmoid bone of rat (Figure by Yuliya Takalchyk-Stukach).

\section{Considering Patients in Clinical Conditions}

It is advisable to inject MSC suspension into soft tissues of upper and partially middle nasal conchae due to high density of olfactory nerve endings $[11,12]$. By the way, we have previously demonstrated that perineural implantation of SC is accompanied with activation of reparative potential of endogenous SC $[8,12]$. Endogenous pools of SC are located in three brain regions: area of olfactory bulbs, hippocampus and brain regions close to ventricles [12]. Unfortunately, endogenous SC lack reparative potential to recover impaired functions of nervous system when neurodestructive processes, Parkinson's and Alzheimer's diseases develop. Cellular technologies can help in these cases, namely perineural implantation of autologous SC from adipose tissue of the patient. These SC can migrate along nerve fibers from periphery to neural networks in brain and spinal cord. SC express various trophic factors on the way of migration, which have positive impact on both functions of neurons and glial cells close to brain trauma and activity of endogenous brain SC [8].

Unfortunately, authors understand that cellular technologies are not always act as panacea - regarding the situation with Stephen Hawking mentioned above. We lack more effective treatment approaches - for example, the ones based on combination of cellular methods with technologies of neural network formation in 3D-space. But this refers to close future.

\section{Conclusion}

\section{Development Prospects of Therapeutic Technologies in Brain Diseases}

There are frequent situations in modern clinical practice which require conditions for penetration of medicinal substances or SC into brain. Selective permeability of blood-brain barrier prevents penetration of cellular elements into cranial cavity (SC, in particular). At the same time, protocols of systemic administration of MSC have been recently developed for treatment of neurodestructive processes. Above mentioned stimulated authors to develop an alternative method which would allow increasing effectiveness of cellular elements and medicinal substances penetration into cranial cavity. It is also necessary to develop such technology which will guarantee advanced penetration of SC but not toxic substances into brain tissue. Scientists who are aimed at widening of cellular technologies area of stem cells use should set sights on solving of mentioned problem and enhancement of developed technology.

\section{Acknowledgement}

This investigation was sponsored by SSTP "New methods of medical care", Section "Transplantation of cells, tissues and organs" (2016-2020), SPSI “Convergence", and by grant 000 "Synergy".

\section{References}

1. Donega V, Nijboer CH, Braccioli L, Slaper-Cortenbach I, Kavelaars A, et al. (2014) Intranasal administration of human MSC for ischemic brain injury in the mouse: In vitro and in vivo neuroregenerative functions. PLoS One 9(11): e112339.

2. Ji G, Liu M, Zhao XF, Liu XY, Guo QL, et al. (2015) NF-кB Signaling is Involved in the Effects of Intranasally Engrafted Human Neural Stem Cells on Neurofunctional Improvements in Neonatal Rat HypoxicIschemic Encephalopathy. CNS Neurosci Ther 21(12): 926-935.

3. Oppliger B, Joerger-Messerli M, Mueller M, Reinhart U, Schneider P, et al. (2016) Intranasal Delivery of Umbilical Cord-Derived Mesenchymal Stem Cells Preserves Myelination in Perinatal Brain Damage. Stem Cells Dev 25(16): 1234-1242.

4. Stokowska A, Atkins AL, Morán J, Pekny T, Bulmer L, et al. (2017) Complement peptide C3a stimulates neural plasticity after experimental brain ischaemia. Brain 140(2): 353-369. 
5. Kulchitsky VA, Shanko YG, Molchanov PG, Cherehkevich SN, Chotianovich MO, et al. (2012) The direction of stem cells movement into the brain depends on the areas of their injection into peripheral parts of the nervous system. Biological Motility: Fundamental and Applied Science. Pushchino. Foton-Vek, pp. 99-101.

6. Stukach Y, Gainutdinov Kh, Dosina M, Pashkevich S, Andrianov V, et al (2016) Migration of neural stem cells in hippocampal slices in hypoxia modeling. J Stem Cells Regen Therapy 12(1): 1-8.

7. Kulchitsky V, Zamaro A, Shanko Y, Koulchitsky S (2018) Positive and negative aspects of cell technologies in cerebral diseases. J Neurol Stroke 8(2): 87-88.

8. Kulchitsky V, Zamaro A, Navitskaya V, Shanko Y, Pashkevich S, et al (2018) Perspectives of stem cells use in alzheimer's disease treatment. J Neurol Stroke 8(3): 190-191.

9. Shanko Y, Zamaro A, Takalchik-Stukach Y, Koulchitsky S, Pashkevich S, et al. (2018) Mechanisms of Neural Network Structures Recovery in Brain Trauma. Biomed J Sci \& Techn Res 7(5): 1-2.

10. Kulchitsky V, Zamaro A, Pashkevich S, Sushko T, Koulchitsky S (2018) Nasolacrimal way of stem cells implantation. J Neurol Stroke 8(2): 110 111.

11. Shanko Y, Navitskaya V, Zamaro A, Krivenko S, Zafranskaya M, et al. (2018) Prospects of Perineural Administration of Autologous Mesenchymal Stem Cells of Adipose Tissue in Patients with Cerebral Infarction. Biomed J Sci \& Tech Res 10(1): 1-3.

12. Shanko Y, Navitskaya V, Zamaro A, Zafranskaya M, Krivenko S, et al. (2018) Somatotopic principle of perineural implantation of stem cells in patients with brain injuries. J Neurol Stroke 8(5): 259-261.

13. Chen S, Singh RJ, Kamal N, Hill MD (2018) Improving care for acute in-hospital ischemic strokes-A narrative review. Int J Stroke $1: 1747493018790029$

14. Chung JW, Park SH, Kim N, Kim WJ, Park JH, et al. (2014) Trial of ORG 10172 in Acute Stroke Treatment (TOAST) classification and vascular territory of ischemic stroke lesions diagnosed by diffusion-weighted imaging. J Am Heart Assoc 3(4): pii: e001119.

15. Doeppner TR, Hermann DM (2014) Stem cell-based treatments against stroke: Observations from human proof-of-concept studies and considerations regarding clinical applicability. Front Cell Neurosci 8: 357.

16. Doeppner TR, Bähr M, Hermann DM, Giebel B (2017) Concise Review: Extracellular Vesicles Overcoming Limitations of Cell Therapies in Ischemic Stroke. Stem Cells Transl Med 6(11): 2044-2052.

17. Kalladka D, Sinden J, Pollock K, Haig C, McLean J, et al. (2016) Human neural stem cells in patients with chronic ischaemic stroke (PISCES): A phase 1, first-in-man study. Lancet 388(10046): 787-796.

18. Qiao LY, Huang FJ, Zhao M, Xie JH, Shi J, et al. (2014) A two-year followup study of cotransplantation with neural stem/progenitor cells and mesenchymal stromal cells in ischemic stroke patients. Cell Transplant 23(1): S65-S72.

19. Xiao J, Nan Z, Motooka Y, Low WC (2005) Transplantation of a novel cell line population of umbilical cord blood stem cells ameliorates neurological deficits associated with ischemic brain injury. Stem Cells Dev 14(6): 722-733.

20. Dhuria SV, Hanson LR, Frey WH 2nd (2010) Intranasal Delivery to the Central Nervous System: Mechanisms and Experimental Considerations. J Pharm Sci 99(4): 1654-1673.

21. Pires A, Fortuna A, Gilberto AG, Falcão A (2009) Intranasal Drug Delivery: How, Why and What for? J Pharm Pharmaceut Sci 12(3): 288-311.

22. Cross DJ, Minoshima S (2013) Perspectives on Assessment of Stem Cell Therapy in Stroke by 18F-FDG PET. J Nucl Med 54(5): 667-669.

23. Daadi MM, Hu S, Klausner J, Li Z, Sofilos M, et al. (2013) Imaging Neural Stem Cell Graft-Induced Structural Repair in Stroke. Cell Transplant 22(5): 881-892.

24. Civelek AC (2016) New Insights: PET Imaging to Document Synergistic Therapy Effect of the "Old," Ancient Recipe and the "New," Modern Drug in Stroke. J Nucl Med 57(4): 499-500.

25. Shichinohe H, Kawabori M, Hiroaki I, Tuyoshi T, Takeo A, et al. (2017) Research on advanced intervention using novel bone marrOW stem cell (RAINBOW): A study protocol for a phase I, open-label, uncontrolled, dose-response trial of autologous bone marrow stromal cell transplantation in patients with acute ischemic stroke. BMC Neurol 17(1): 179 .

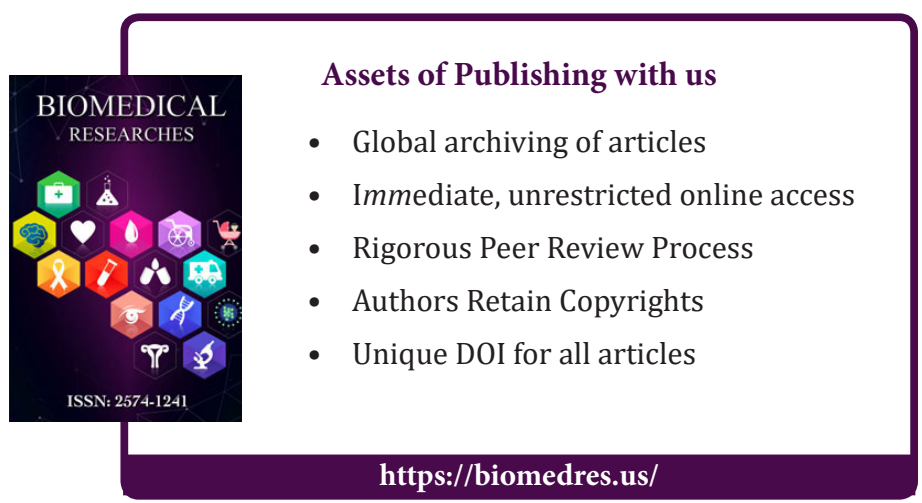

tips://biomedres.us/
ISSN: 2574-1241

DOI: $10.26717 / B J S T R .2018 .10 .001959$

Kulchitsky Vladimir. Biomed J Sci \& Tech Res

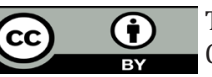

This work is licensed under Creative

Commons Attribution 4.0 License

Submission Link: https://biomedres.us/submit-manuscript.php 